\title{
Giving Advice in Negotiation using Physiological Information
}

\author{
Masahide YUASA, Yoshiaki YASUMURA, Katsumi NITTA \\ Dept. of Computational Intelligence and Systems Science \\ Tokyo Institute of Technology, Japan
}

\begin{abstract}
In this paper, we propose a method for giving advice to participants of negotiation using physiological information. We apply Newcomb's A-B-X model as a model human relation to negotiation. Based on the model, we adopt the user's preference of a proposal, an impression of opponent's attitude and a degree of the user's emotional disturbance in the generating advice tool. We measure the user's perspiration and pulse rate to extract the user's emotional disturbance. The tool learns the relation between the user's preferences and the advice. The experimental results show that the tool can improve the quality of advice.
\end{abstract}

\section{Introduction}

Negotiation is a process to reach an agreement between participants who have different goals by exchanging proposals. In order to negotiate efficiently, several software tools to support negotiation through Internet have been developed. Most of these tools use the utility value to select a proposal.

However, we often decide the next proposal based on various kinds of factors. For example, when we negotiate about compensation of a car accident, we evaluate proposals by considering not only the utility value but also the feeling about the opponent and the emotional disturbance.

Therefore, we use new psychological parameters such as a user's emotional disturbances and an impression of opponent's attitude, to select suitable proposals. The user's emotional disturbance is extracted by using perspiration and pulse rate.

In this paper, we will describe the method to give the users advice by using physiological parameters and based on the Newcomb's model for human relations. Section 2 introduces Newcomb's model in negotiation. Section 3 describes an overview of a negotiation support system by using physiological information.
Section 4 describes the evaluation of the system. Finally, Section 5 summarizes our research.

\section{Human Relation in Negotiation}

We introduce Newcomb's A-B-X model [3], which represent the human relation in psychology. Then we explain the process of negotiation by using this model.

\subsection{Newcomb's A-B-X Model}

A-B-X model consists of two persons, labeled A and B, and one impersonal entity, which could be a physical object or an idea, labeled $X$. The model represents the person's cognitive structure of relations among A, B and $X$. The link sign is plus $(+)$ when a person has positive feeling and the link sign is minus $(-)$ when a person has negative feeling for one of these entities. There are 16 states of the relation between A, B and X, which are classified into balanced one and unbalanced one. Figure 1 illustrates examples of these states. The unbalanced states are unstable and tend to move to the balanced states.

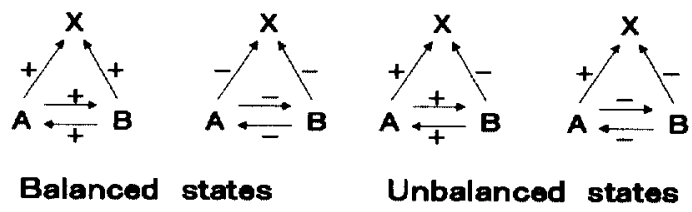

Figure 1. Balanced states and unbalanced states

\subsection{The A-B-X model in Negotiation}

To describe psychological relation among participants and proposals of negotiation, we consider $A$ and $B$ as participants, and $X$ as a current proposal. The link from A to B means A's impression of B's attitude and 
the link $\mathrm{A}$ to $\mathrm{X}$ means A's preference of $\mathrm{X}$.

Figure 2 shows state transition in negotiation. When $A$ offers a proposal $\mathrm{X}$ and $\mathrm{B}$ doesn't agree with $\mathrm{X}$, the link from $A$ to $X$ is plus and the link from $B$ to $X$ is minus. Because A's preference of $X$ is different from $B$ 's, they have the negative feelings each other. This state is represented as the state (1) in Figure 2.

Then B offers a new proposal but A doesn't agree with it. It is the state (2).By offering new proposals in turn, state changes from (1) to (2) and from (2) to (1). If they agree with a certain proposal, then the state move to (4). Because this state is unbalanced, the state move to (6). This state means that they reach an agreement. However, there are the other routes to reach an agreement. For example, if $\mathrm{A}$ changes the attitude in the state (1), then the state will be the state (5). By the change of A's attitude, B may change the preference of the proposal, and the state will be the state (6).

In this way, there are two types of route to reach an agreement, one is the change the preference of proposals, and the other is the change of an impression of the attitude. The preference of proposals and the impression of the attitude are affected by the emotional disturbance. Therefore, we adopt the following parameters in our negotiation support tool.

1) Preference of a proposal

2) Impression of an opponent's attitude

3) User's emotional disturbance

\section{An Overview of Negotiation Support System}

\subsection{Functions of the System}

A user negotiates with an opponent through the computer network in our negotiation support system. The system has two tools, the generating advice tool and a video and audio conferencing tool, as in Figure 3. The user inputs a preference of a proposal and an impression of an opponent's attitude, before offering a proposal. At the same time, the user's physiological information is measured by sensors. Then the generating advice tool gives the user advice from the preference, the impression and the user's emotional disturbance. The user can offer the proposals by referring to the advice with the conferencing tool. Then the user evaluates the advice and the evaluation is used to select next advice.

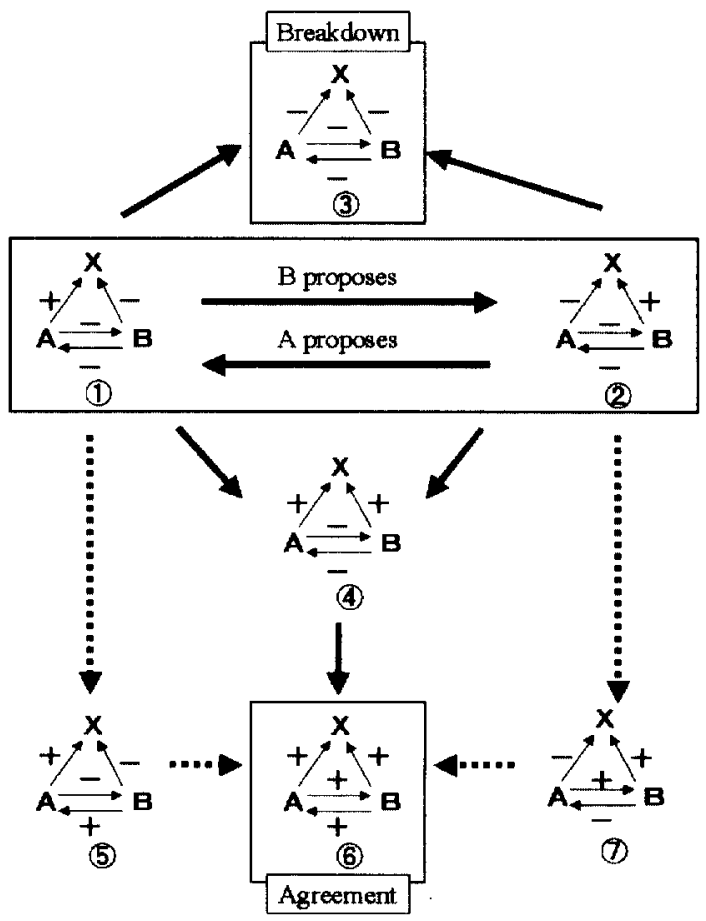

Figure 2. State trangition in negotiation

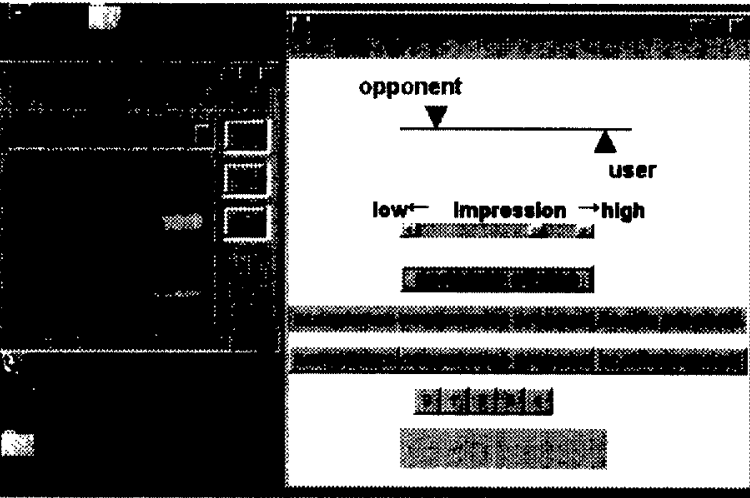

Figure 3. A video and audio conferencing tool and negotiation support tool 


\subsection{Architecture of the System}

The system consists of the conferencing tool and the generating advice tool, as in Figure 4. The conferencing tool enables us to use image and voice with camera and microphone and hold face-to-face conversations with an opponent in negotiation. And the generating advice tool consists of the 3 modules, a dialog box module, an extracting emotional disturbance module, and a generating advice module.

1) Dialog Box Module

This module shows a dialog box and receives the user's input; an impression of opponent's attitude and a preference of proposal. The user evaluates these values from image and voice subjectively using the conferencing tool. These parameters are sent to Generating Advice Module. And the dialog window shows advice given from Generating Advice Module. Then the user evaluates the advice and the evaluation is also sent to Generating Advice Module.

2) Extracting Emotional Disturbance Module

This module receives physiological information such as pulse rate and perspiration from sensors. And this module learns the relation between the user's emotional disturbance and the sensor data by using Probabilistic Neural Networks (PNN)[4][5]. The information is sent to Generating Advice Module.

\section{3) Generating Advice Module}

This module generates advice using a preference of a proposal, an impression of opponent's attitude and the user's emotional disturbance. The advices are classified into three types: acceptance, compromise proposal and hostile proposal. In order to provide information suitable for the individual preferences of user, the system learns preference data by PNN.

\subsection{Extracting Emotional Disturbance}

The user's emotional disturbance is measured by the following sensors.

1) Perspiration sensor

We use 'Perspiro201' by Suzuken as a perspiration sensor as in Fig 5. This sensor measures mental sweating, which occurs hands or thumb by emotional disturbances.

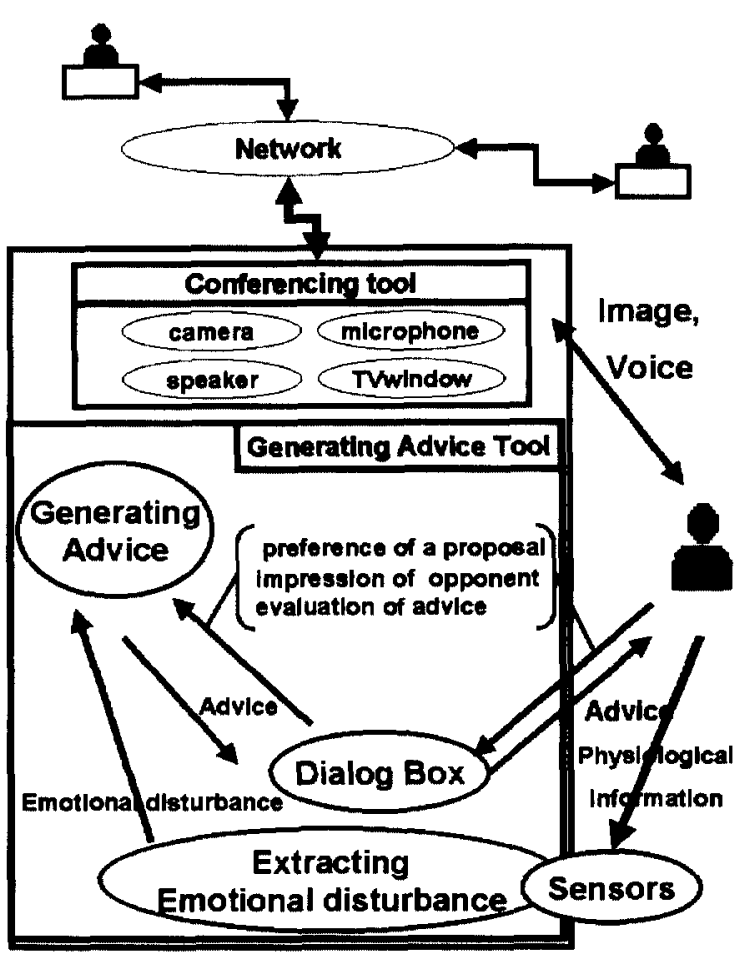

Figure 4. Architecture of System

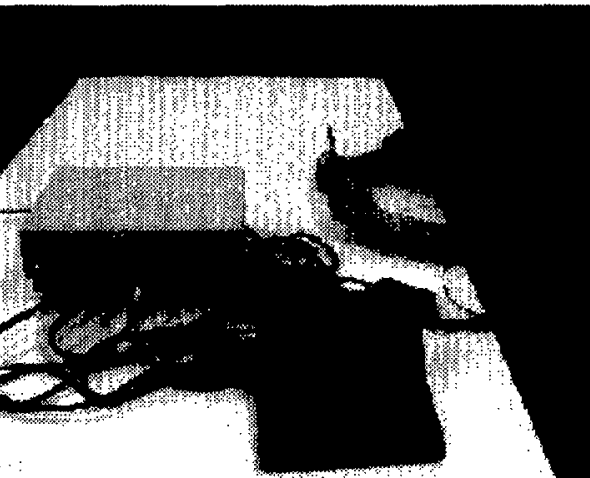

Figure 5. Measuring equipment

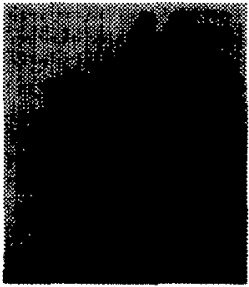

Figure 6. Perspiration sensor 
The mental sweating occurs in response to excitement, fear, anxiety, and a multitude of other disturbed states.

\section{2) Pulse rate sensor}

We use the pulse rate sensor made of infrared sensor as in Fig 7. Generally, it's well known that emotional disturbances quicken the pulse rate.

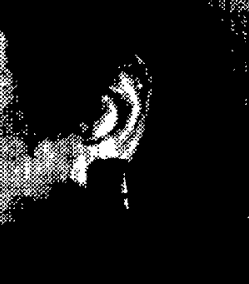

Figure 7. Pulse rate sensor

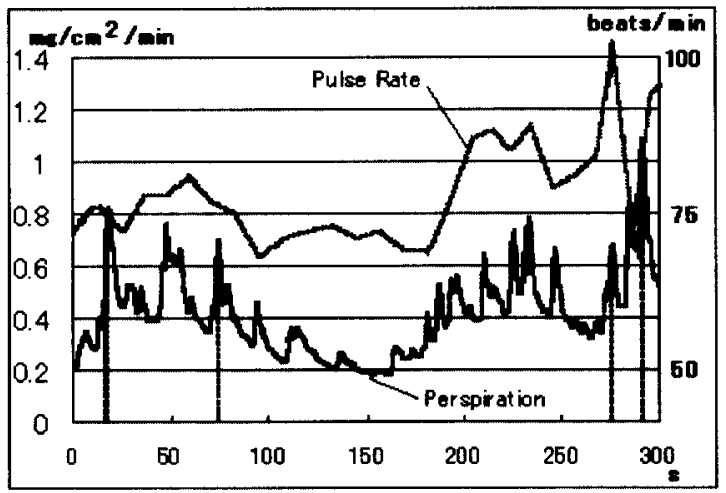

Figure 8. Perspiration and pulse rate in negotiation

Figure 8 shows values of pulse rate and perspiration in a example of negotiation [6]. The horizontal axis means time, the vertical axis means sensor values each time. In this example, the status of negotiation changed as follows. They exchanged the opinions initially. However, his standpoint becomes worse gradually. Though he tried to improve his standpoint, he failed.

His emotional disturbance reflected to sensor value. As the results, we can see the following properties:

1) Perspiration is sensitive to the emotional disturbance. However, because the mental sweating occurs in response to the multitude of other factors: talking, thinking deeply and so on, it's difficult to discriminate a psychological reason from the other reasons.

2) The pulse rate goes up by the emotional disturbance without the noise. However, it is slow to react to the disturbance and not more sensitive than perspiration.

Considering these properties, we calculate a level of the emotional disturbance from the following three values:

1) The difference of value of the amount of perspiration

The emotional disturbance agrees well with the peak of perspiration, which shows the broken line.

2) The moving average value of the amount of perspiration

The shift of the amount of perspiration indicates the emotional disturbance approximately.

3) The difference of pulse rate

The pulse rate also indicates the emotional disturbance approximately.

It's difficult for us to find the relation among the values and the emotional disturbance. And the relation changes for each. Therefore, we use PNN to learn the relation. PNN can learn data cumulatively and quickly. PNN is suitable for the classification [7]. The objective evaluation of a degree of user's emotional disturbance by subject is used as the supervised signal.

\subsection{Generating Advice}

The generating advice tool makes advice from an impression of opponent's attitude, a preference of a proposal and the user's emotional disturbance. The tool gives the user one of three advices: acceptance, compromise proposal and hostile proposal. The user can negotiate by referring to the advice.

And we should consider differences among individuals. Even if one person likes certain advice, another person may like different one. Therefore, the tool learns the preference by PNN. The user's preference of advice is used as the supervised signal. Since the user utilize the negotiation support tool, the user needs to input test patterns in advance. Figure 9 describes one of results 
of learning training data. $\mathrm{X}$-axis, $\mathrm{Y}$-axis and $\mathrm{Z}$-axis means the user's preference of a proposal, the impression of an opponent's attitude and the user's emotional disturbance.

Figure 10 and 11 are a cutting plane by $Z$-axis for two different persons, $A$ and $B$. As we can see these figures, the advice person wants are affected by the impression of the opponent's attitude and the user's emotional disturbance. It's mean that even if a person's preference of a proposal is low, the good relation between the person and the opponent cause acceptance of the person. And by comparing figure 10 and 11 , we find that the differences among individual persons.

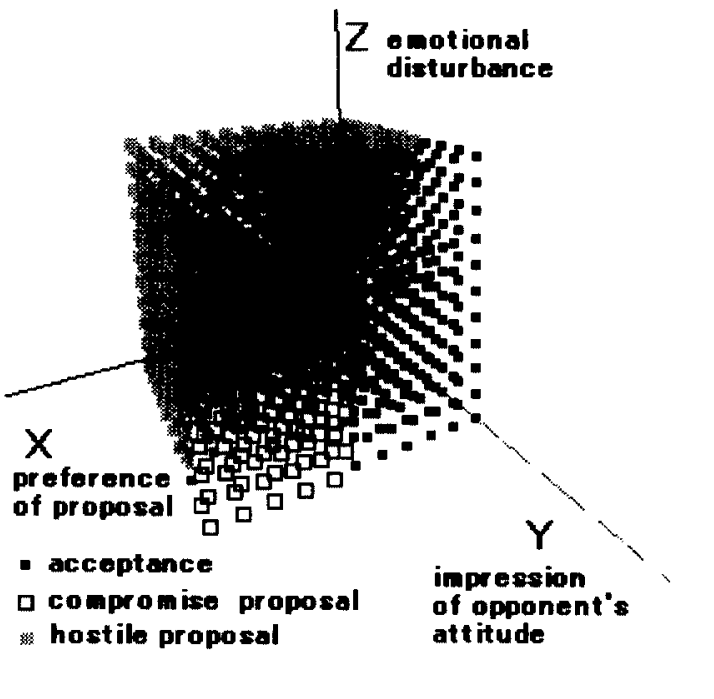

Figure 9. A distribution of advice

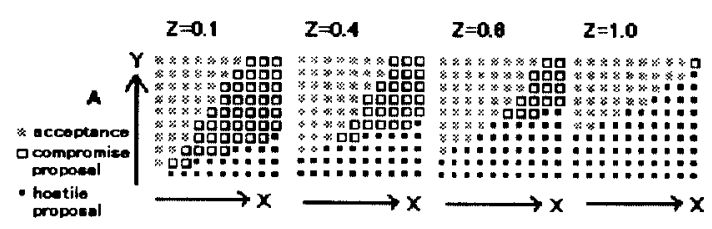

Figure 10. Cutting plane by Z-axis (person A)

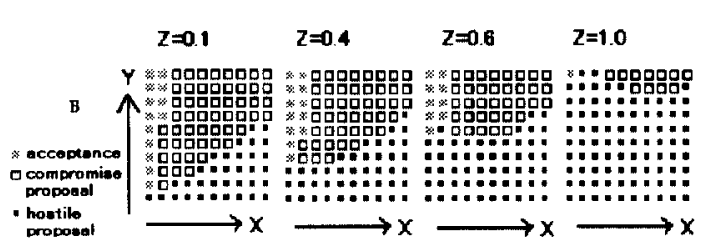

Figure 11. Cutting plane by Z-axis (person B)

\section{Evaluation of Advice}

We evaluate the effect of the system by comparing with two advices. One is the advice generated from only the preference of proposals. Another is the advice generated from the preference, an impression of opponent and emotional disturbance. The results of the selection are shown in Table 1 . The each column value means 'support rate';

support rate $=\frac{\text { the number of advice which the user selects }}{\text { the number of advice }}$

\begin{tabular}{|l|r|r|}
\hline & only preference & our tool \\
\hline acceptance & $14 / 29$ & $6 / 9$ \\
\hline compromise proposal & $16 / 26$ & $21 / 42$ \\
\hline hostile proposal & $21 / 34$ & $30 / 38$ \\
\hline & $51 / 89$ & $57 / 89$ \\
\hline
\end{tabular}

Table 1. Compare with two methods

As we can see from Table 1, the tool that used only preference matched the user's move by $57 \%$, our tool matched by $64 \%$.

The results mean that the user makes decisions by not only preference of proposals but also an impression of opponent's attitude and an emotional disturbance. One of the reasons that advice doesn't satisfy the user is that our system failed to extract proper emotional disturbance.

\section{Conclusion}

We proposed a method for giving advice to users in negotiation using physiological information.

We applied A-B-X model as a model of human relation to negotiation. From the model, we decided to 
consider a preference of a proposal, an impression of opponent's attitude and the user's emotional disturbance. In order to extract the user's emotional disturbance, we measured perspiration and pulse rate as physiological information. Considering these results, we made the negotiation support tool. The experimental results show that our tool can improve the quality of advice by considering these parameters. However, there is a problem that the user must input a preference of proposals and an impression of opponent's attitude by the user's manual operations. It's desired to improve the feature integrates user's data automatically.

In this report, we use perspiration and pulse rate in order to measure the emotional disturbance. We may extract it from another sensors. By using the additional information, the advice can be more suitability and detailed.

\section{References}

[1] Katsumi Nitta, Akira Yamazaki, Takashi Kitou and Yoshiaki Yasumura, A Negotiation Support Tool using a Computer Network, Technical report of IEICE, OFS98-19, AI98-28, 1998, (in Japanese)

[2] AkiraYamazaki, Takashi Kitou, Yoshiaki Yasumura and Katsumi Nitta, On an Evaluation Method for Next Moves of a Negotiation Support System, Report of IPSJ, Intelligence and complex systems, 114-6, 1998, (in Japanese)

[3] Isamu Saito, The Handbook of Experimental Social Psychology 2: Interpersonal Attraction and Social Motives, Seishin Shobo, 1987, (in Japanese)

[4] DONALD F.SPECHT : Probabilistic Neural Networks, Neural Networks, Vol3, pp.109-118, 1990

[5] J.P.Bigus, Data Mining with Neural networks, The McGraw-Hill Companies, 1996.

[6] Masahide Yuasa, Yoshiaki Yasumura and Katsumi Nitta, Analysis of Physiological Information in Negotiation, SIG-FAI-9903-13, 1999, (in Japanese)

[7] PHILIP.D.WASSERMAN, Advanced Methods in Neural Computing, A Division of Wadsworth, Inc., 1993 